\title{
Artefacts in continuous overnight blood pressure assessment based on pulse transit time
}

\begin{abstract}
Continuous non-invasive blood pressure measurements bear a high potential. Particular in Somnology they allow to derive comfortably the systolic and diastolic blood pressure from an electrocardiogram and a synchronous photoplethysmogram without sleep disruption. In this short article some possible problems of this method are discussed along overnight recordings with a S OMNOtouch NIBP device.
\end{abstract}

Keywords: Electrocardiogram ECG, Photoplethysmogram PPG, Pulse Transit Time PTT, Pulse Wave Velocity PWV, Photoplethysmogram Intensity Ratio PIR, SOMNOtouch ${ }^{\mathrm{TM}}$.

https://doi.org/10.1515/cdbme-2021-2215

\section{Introduction}

Blood pressure (BP) is an important measure for risk assessment of cardiovascular diseases. In particular, home based measurements have shown to be a key [1]. An important feature in a normal diurnal course of the BP is the so-called dipping, the reduction of the BP during night-time [2, 3].

Indirect BP measurement has a long history [4], the standard cuff-based auscultatory method was introduced in 1905 by N. S. Korotkoff (sometimes transliterated as Korotkov). So it is surprising that the basic theoretical works from D. J. Korteweg and A. I. Moens are more than twenty years earlier, from 1878 $[5,6]$. Although their outcomes differ [7], they are commonly cited together using the formula

$$
c=\sqrt{\frac{E \cdot h}{\rho \cdot D}},
$$

*Corresponding author: Dagmar Krefting: UMG Göttingen, Department of Medical Informatics, Göttingen, Germany, and HTW Berlin, Berlin, Germany, dagmar.krefting@med.unigoettingen.de Henning Dathe and Tibor Kesztyüs: UMG Göttingen, Department of Medical Informatics, Göttingen, Germany wherin $c$ is the speed of the fluid, $\rho$ its density, $E$ the elastic modulus of the vessel's wall, $h$ its thickness and $D$ the interior diameter of the vessel. Nowadays, the velocity $c$ is identified with the pulse wave velocity, usually abbreviated as $p w v$. The pulse transit time $P T T$ is achieved by dividing a characteristic individual distance $L$ from the heart to the fingertip, where the PPG is usually measured, through the $p w v$

$$
P T T=\frac{L}{p w v} \text {. }
$$

This time is measured typically as the time between the REvent of the ECG and the increasing inflection point of the PPG. By assuming constant proportions in humans, $L$ may be derived from the individual body height $L_{B}$ by approximating $L=k \cdot L_{B}$. with an appropriate constant $k$. Since $h, \rho$ and $D$ are assumed to be individual and more or less constant quantities, we seek a r elationship between $E$ and the systolic blood pressure $p$ (usually abbreviated as $S B P$ ).

By examining dogs, D. J. Hughes et al. found 1979 the empirical relationship [8]

$$
E=E_{0} \exp (a \cdot p)
$$

This would, inserted to Eq. (1), lead to a result of the type $c=c_{0} \cdot \exp (a \cdot p / 2)$. On the other hand, Chen et al. derived in 2009 [9]

$$
p=b \exp \left(-\frac{k}{c}\right),
$$

in which the coefficients $b$ and $k$ depend on age and sex. So there might be no established relationship between $p$ and $c$.

In the last step, the diastolic blood pressure $D B P$ has to be estimated. By taking into account the Photoplethysmogram Intensity Ratio pir, X. R. Ding et al. [10] derived 2016 a relationship of the form $D B P=a / P T T+b / p^{2}{ }^{2}$, where $a$ and $b$ are individual constants and pir reflects the influence of the varying vessel's diameter $D$.

\subsection{The SOMNOtouch NIBP device}

The SOMNOtouch ${ }^{\mathrm{TM}}$ NIBP (ST-BP) is a cer tified medical device for outpatient home sleep testing including continuous noninvasive BP measurement based on pulse transit time. As conventional long-term measurements with BP cuffs typically disrupt sleep, the device bears high potential for realistic BP 
measurements during sleep. Details on the algorithm used can be found in H. Gesche et al. 2012 [11] and the corresponding patents [12]. The $p w v$ therein is calculated from the $p t t$ using body height $L_{B}$. The relationship between $p w v$ and $B P$ is assumed as

$$
B P_{P T T}=P 1 \cdot p w v \cdot \exp (P 3 \cdot p w v)+P 2 \cdot p w v^{P 4}-
$$$$
\left(B P_{P T T, c a l}-B P_{c a l}\right) \text {, }
$$

where the $p w v$ is measured in $\mathrm{cm} / \mathrm{ms}$ and the $B P$ in $m m H g$. The coefficients $P 1$ through $P 4$ are determined by a least square fitting procedure to the values given as $P 1=700, P 2=766000$, $P 3=-1, P 4=9[11,12]$. The terms at the end of the formula are determined by an individual calibration using a cuff-based method. Its accuracy according to the European Society of Hypertension International Protocol (ESH-IP) revision 2010 criteria [13] has been stated 2010 in [14]. However, very recent comparisons of the 24-hour BP measurements obtained with ST-BP have resulted in poor agreement. But contradictory results have been published 2021 in $[15,16]$. Both studies have conduced simultaneous measurements with ST-BP and a cuffbased BP monitors as reference (Spacelabs 90217A and 90217), with 51 patient with essential hypertension [15] and 71 subjects, including 28 patients with antihypertensive treatment and $43 \mathrm{~h}$ ealthy volunteers [16]. Both found significant increase of the mean SBP and DBP measured with ST-BP compared with the reference devices. Interestingly, in particular the nighttime measurements show large deviations, with a mean SBD of about $15 \mathrm{mmHg}$ higher with ST-BP. A new software version (Version 1.5) reduced the difference to about $11 \mathrm{mmHg}$ higher. Both studies report that the night-time BP dipping is not visible in ST-BP in many of the cases where it is obtained in the reference; and state that the device is not suitable for assessment of night-time BP. It has also been suspected that in particular extreme BP values are less in agreement with the reference while the event number was not sufficient for statistical analysis. In summary, the current data situation raises concerns on the reliability of ST-BP for continuous overnight measurements.

\section{Material and Methods}

Whole-night measurements with the SOMNOtouch ${ }^{\mathrm{TM}}$ device on volunteers are carried out. BP calibration is performed at the beginning and the end of the night using a cuff-based method at the right arm in a semi-recumbent position. For the analysis in this short paper, data of 4 different nights from one female volunteer with treated essential

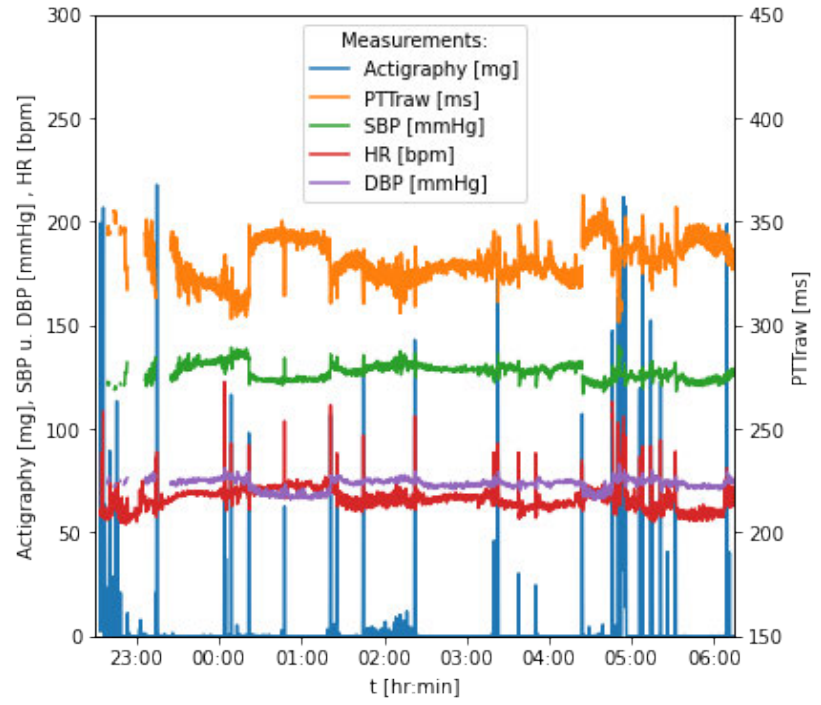

Figure 0: Course of the different signals provided by ST-BP during one night. For better visibility, the PTT signal has been reduced by an offset of $150 \mathrm{~ms}$. HR: heart rate, SBP/DBP: systolic/diastolic blood pressure, PTTraw: pulse transit time annotated as "raw" by ST-BP.

hypertension (age 49) have been included. Earlier conventional long-term measurements on BP of this subject have shown night-time BP dipping. Further nights and measurements of other volunteers have been excluded, as they have been recorded before a firmware update of the Somnotouch device, and BP data cannot be exported as files. The data have been exported with vendor's recommended software domino light, version 1.5. The actigraph signals come from the wrist-worn SOMNOtouch device on the left hand. The PPG signals are recorded also at the same hand. At the beginning of one night, the subject put herself in different body positions and different positions of the left arm, where the device is put on, in the following order: back, left side with arm stretched down, left side with arm angled and up (hand put under the pillow), right side with arm stretched down, right side with arm angled and up (the hand under the pillow). The data has been imported and visualized with Python, version 3 , the corresponding notebooks and data are available upon request. The data is analysed visually regarding artefacts and unplausible BP values.

\section{Results}

The derived BP shows a high variability during the night with notable jumps that lead to different SBP plateaus. Figure 1 shows different ST-BP signals of one of the measurements during the full night. Typical movement artefacts are seen in line with the actigraphic signal (blue line), Most of them are 


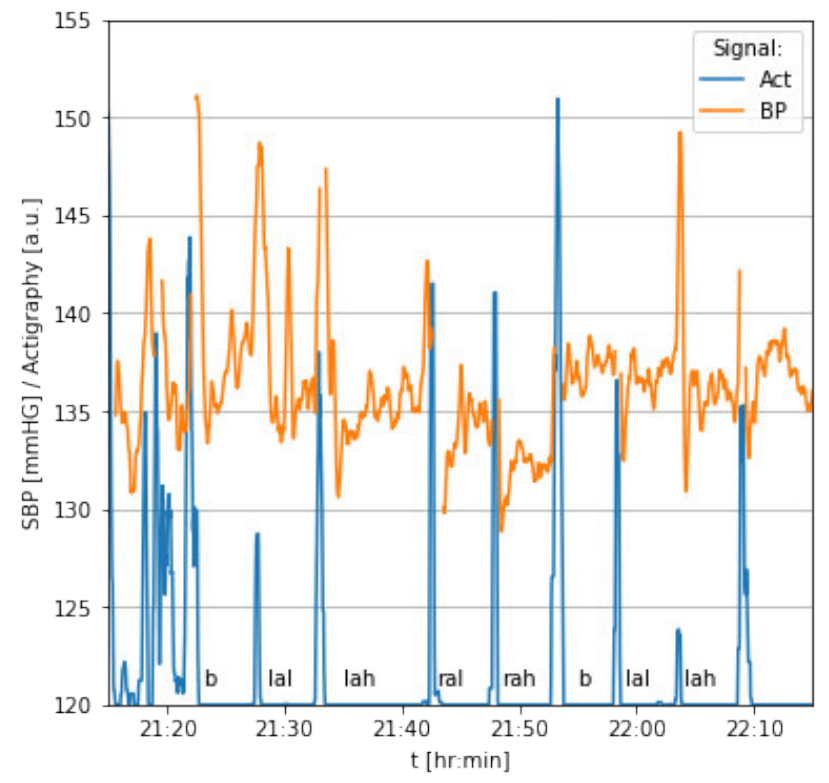

Figure 2: Course of the different signals provided by ST-BP during one night. For better visibility, the PTT signal has been reduced by an offset of $150 \mathrm{~ms}$. SBP/DBP: systolic/diastolic blood pressure, PTTraw: pulse transit time indicated as "raw" by ST-BP. b: back, I/ral: lying on the left/right side arm stretched towards the knee (low), l/rah: lying on the left/right side arm angled with hand below the pillow (high).

short term artefacts, but at around 0:20 a.m. the PTT (yellow line) stays at higher level that leads to an abrupt decrease in SBP (green line) and -less pronounced-in DBP (violet line). After about one hour, another movement is observed that leads again to an inverse signal jump in the PTT and consequently to higher plateaus in the BP signals. The same phenomenon is observed at about 4:20 a.m. The indicated body position is reported as back position until the actigraphic signal at 4:20 a.m., where standing is reported, while wake stage is indicated from 4:50 a.m. to 5:10 a.m. A patient marker indicating that the subject is awake and able to handle the device has been set at 4:55 a.m. Different body positions have been taken according to the protocol described in Methods for 5 minutes (one period is longer as the timer stopped meanwhile). The reported body position by ST-BP is always back position, twice during the measurement a short standing position period is reported when the position was changed. The resulting SBP is shown in Figure 2. While there is no difference visible in the left position independent on arm position and right side with arm low, the visual inspection indicates a $\mathrm{n}$ otable lower plateau in right position with hand below the pillow (rah) and a higher SBP in back position (b) with a difference between rah and $b$ of about $5 \mathrm{mmHg}$.

\section{Discussion}

Visual inspection revealed some at least unexpected SBP variations during night time. Synchronously with the actigraphic signal, the PTT changes abruptly to a higher plateau, jumping partially back about $1 \mathrm{hr}$ later, also accompanied by movement detection. A possible event might be the change of body position. Qualitative assessment of the influence of body position on the SBP support this assumption. So far, we could not find a description of such an effect in the literature, so further investigation is required. An increase in arterial stiffness and therefore in carotid-femoral pulse wave velocity has been observed for $45 \%$ heads up and Fowlers position, where only the upper body is lifted [17]. If such behaviour is also effective in the blood vessels involved in the PTT measurement, this might explain an overall lower PTT and consequently higher BP during the night, if the calibration is performed in sitting or standing position.

The dependence of SBP on PTT is modelled in [11, 12] containing an additional term with the PTT to the 9th exponent in the denominator. During episodes of short PTT, due, for instance, to the influence of signal quality on the PTT detection algorithm or clinical reasons, this may result in an inappropriate high BP estimate. This would be in accordance with the impression of Nyvad et al., that in particular extreme values show lower accordance with cuff-based reference measurements [15], and would explain an overall overestimation of the BP. However, the vendor has changed the algorithms in a new software (and presumably firmware) version, that has reduced this overestimation. However, no information could be found about the algorithmic modifications to the published methods. But it shows that metadata such as the software version are utterly important for the interpretation and comparison of biosignal recordings. A more comprehensive study with focus on signal alterations during sleep is currently planned to test the hypotheses obtained by this exploratory analysis.

\section{Author Statement}

Research funding: The authors state no funding involved. Conflict of interest: Authors state no conflict of interest. Informed consent: Informed consent has been obtained from all individuals included in this study. Ethical approval: The research related to human use complies with all the relevant national regulations, institutional policies and was performed in accordance with the tenets of the Helsinki Declaration. 


\section{References}

[1] Pickering TG, Miller NH, Ogedegbe G, Krakoff LR, Artinian NT, Goff D. Call to action on use and reimbursement for home blood pressure monitoring: Executive Summary. A joint scientific statement from the American Heart Association, American Society of Hypertension, and Preventive Cardiovascular Nurses Association. J Clin Hypertens (Greenwich) 2008;10:467-476. https://doi.org/10.1111/i.1751-7176.2008.08418.x

[2] Stanton A, O'Brien E. Noninvasive 24 hour ambulatory blood pressure monitoring: current status. Postgrad Med J 1993;69: 255-267.

[3] Salles GF, Reboldi G, Fagard RH, Cardoso, CRL, Pierdomenico, SD, Verdecchia, P, Eguchi, K, Kario, K, Hoshide, S, Polonia, J, de la Sierra, A, Hermida, RC, Dolan, E, O'Brien, E, Roush, GC, ABC-H Investigators. Prognostic Effect of the Nocturnal Blood Pressure Fall in Hypertensive Patients: The Ambulatory Blood Pressure Collaboration in Patients With Hypertension (ABC-H) Meta-Analysis. Hypertension 2016;67: 693-700. https://doi.org/10.1161/HYPERTENSIONAHA.115.06981

[4] O'Brien, E \& Fitzgerald, D. The history of indirect blood pressure measurement. In: Birkenhager WH, Keid JL (series eds) Handbook of Hypertension. Vol.14, O'Brien E, O'Malley $\mathrm{K}$ (eds). Blood Pressure Measurement, Amsterdam, Elsevier; 1991: 1-54.

[5] Korteweg, DJ. Ueber die Fortpflanzungsgeschwindigkeit des Schalles in elastischen Röhren, 1878, https://doi.org/10.1002/andp.18782411206.

[6] Moens, Al. Die Pulscurve, Leiden, Brill, 1878.

[7] Tijsseling, AS, Anderson, AA. Isebree Moens and D. J. Korteweg: on the speed of propagation of waves in elastic tubes. Proceedings of the 11th International Conference on Pressure Surges (Lisbon, Portugal, October 24-26, 2012). ISBN 978-1-85598-133-1

[8] Hughes DJ, Babbs CE, Geddes LA and Bourland JD. Measurement of Young's modulus of elasticity of the canine aorta with ultrasound, Ultrasonic Imaging, 1979;1:356-367.

[9] Chen Y, Wen C, Tao G, Bi M and LI G. Continuous and Noninvasive Blood Pressure Measurement: A Novel Modeling Methodology of the Relationship between Blood
Pressure and Pulse Wave Velocity. Annals of Biomedical Engineering, 2009; Vol 37, No 11:2222-2233, https://doi.org/10.1007/s10439-009-9759-1

[10] Ding XR, Zhang YT, Liu J, Dai WX and Tsang HK. Continuous Cuffless Blood Pressure Estimation using Pulse Transit Time and Photoplethysmogram Intensity Ratio. IEEE Trans Biomed Eng, 2016;63:5

[11] Gesche H, Grosskurth D, Küchler K, Patzak A. Continuous blood pressure measurement by using the pulse transit time: comparison to a cuff-based method. Eur J Appl Physiol, 2012;112:309-315, https://doi.org/10.1007/s00421-0111983-3

[12] Patentfamily DE102005014048B4, EP000001704820B1, US20060217616A1 and more.

[13] O’Brien E, Atkins N, Stergiou G, Karpettas N, Parati G, Asmar R, et al. European Society of Hypertension International Protocol revision 2010 for the validation of blood pressure measuring devices in adults. Blood Press Monit 2010;15:23-38.

[14] Bilo G, Zorzia C, Ochoa Muneraa JE, Torlascoa C, Giulia V and Paratia G: Validation of the Somnotouch-NIBP noninvasive continuous blood pressure monitor according to the European Society of Hypertension International Protocol revision 2010; Blood Press Monit 2015; Vol 20 No 5; https://doi.org/10.1097/MBP.0000000000000124

[15] Nyvad J, Christensen KL, Buus NH, Reinhard M: The cuffless SOMNOtouch NIBP device shows poor agreement with a validated oscillometric device during 24-h ambulatory blood pressure monitoring; J Clin Hypertens. 2021;23:61-70; https://doi.org/10.1111/ich.14135

[16] Socrates, T, Krisai, P, Vischer, AS, Meienberg, A, Mayr, M, Burkard, T. Improved agreement and diagnostic accuracy of a cuffless 24-h blood pressure measurement device in clinical practice. Sci Rep 2021;11:1143. https://doi.org/10.1038/s41598-020-80905-x

[17] Cohen, J, Pignanelli, C, Burr, J. The Effect of Body Position on Measures of Arterial Stiffness in Humans. J Vasc Res, 2020;57:143-151. https://doi.org/10.1159/000506351 